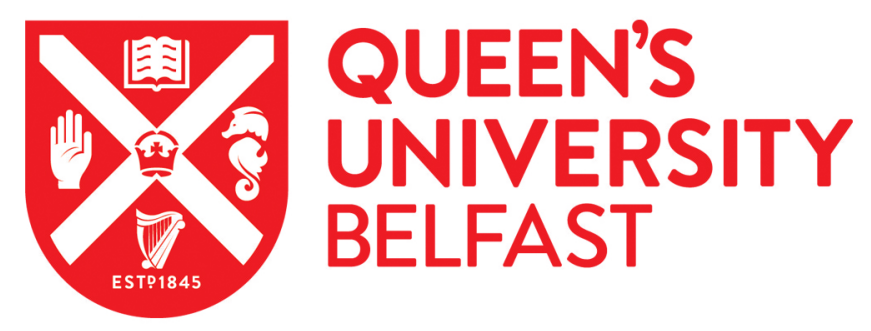

\title{
A comparison of inferential analysis methods for multilevel studies: Implications for drawing 2 conclusions in animal welfare science
}

Stevens, K. N., Asher, L., Griffin, K., Friel, M., O'Connell, N., \& Collins, L. (2017). A comparison of inferential analysis methods for multilevel studies: Implications for drawing 2 conclusions in animal welfare science. Applied Animal Behaviour Science. https://doi.org/10.1016/j.applanim.2017.08.002

Published in:

Applied Animal Behaviour Science

Document Version:

Peer reviewed version

Queen's University Belfast - Research Portal:

Link to publication record in Queen's University Belfast Research Portal

\section{Publisher rights}

Copyright 2017 Elsevier.

This manuscript is distributed under a Creative Commons Attribution-NonCommercial-NoDerivs License

(https://creativecommons.org/licenses/by-nc-nd/4.0/), which permits distribution and reproduction for non-commercial purposes, provided the author and source are cited.

\section{General rights}

Copyright for the publications made accessible via the Queen's University Belfast Research Portal is retained by the author(s) and / or other copyright owners and it is a condition of accessing these publications that users recognise and abide by the legal requirements associated with these rights.

Take down policy

The Research Portal is Queen's institutional repository that provides access to Queen's research output. Every effort has been made to ensure that content in the Research Portal does not infringe any person's rights, or applicable UK laws. If you discover content in the Research Portal that you believe breaches copyright or violates any law, please contact openaccess@qub.ac.uk. 
4 Kara N. Stevens ${ }^{1,2}$, Lucy Asher ${ }^{3}$, Kym Griffin $^{4,5}$, Mary Friel ${ }^{4,6}$, Niamh O'Connell ${ }^{4}$ and Lisa M.

5 Collins ${ }^{1,6}$

6

7

$8 \quad{ }^{1}$ School of Life Sciences, University of Lincoln, Brayford Pool, Lincoln, LN6 7DL

$9 \quad{ }^{2}$ Medical Statistics, Plymouth University Peninsula Schools of Medicine and Dentistry, PL6 8BX

$10{ }^{3}$ Centre of Behaviour and Evolution, Institute of Neuroscience, Newcastle University, NE2 4HH

$11{ }^{4}$ Institute for Global Food Security, Queen's University Belfast, Belfast, BT9 5BN

$12{ }^{5}$ School of Animal, Rural and Environmental Sciences, Nottingham Trent University, NG25 0QF

$13{ }^{6}$ Faculty of Biological Sciences, University of Leeds, Leeds, LS2 9JT

15 Corresponding author: Lisa M. Collins

16 Address: Faculty of Biological Sciences, University of Leeds, Leeds, LS2 9JT

17 Telephone: 00441133435940

18 Email: L.Collins@leeds.ac.uk

19 Running head: Model selection for multilevel repeated measures 
Investigations comparing the behaviour and welfare of animals in different environments have led to mixed and often conflicting results. These could arise from genuine differences in welfare, poor validity of indicators, low statistical power, publication bias, or inappropriate statistical analysis. Our aim was to investigate the effects of using four approaches for inferential analysis of datasets of varying size on model outcomes and potential conclusions. We considered aggression in 864 growing pigs over six weeks as measured by ear and body injury score and relationships with: less and more enriched environments, pig's relative weight, and sex. Pigs were housed in groups of 18 in one of four pens, replicating the experiment 12 times. We applied four inferential models that either used a summary statistic approach, or else fully or partially accounted for complexities in study design. We tested models using both the full dataset $(n=864)$ and also using small sample sizes $(n=72)$.

The most appropriate inferential model was a mixed effects, repeated measures model to compare ear and body score. Statistical models that did not account for the correlation between repeated measures and/or the random effects from replications and pens led to spurious associations between environmental factors and indicators of aggression, which were not supported by the initial exploratory analysis. For analyses on smaller datasets $(n=72)$, due to the effect size and number of independent factors, there was insufficient power to determine statistically significant associations.

37 Based on the mixed effects, repeated measures models, higher body injury scores were associated with more enrichment (coef. est. $=0.09, \mathrm{p}=0.02$ ); weight (coef. est. $=0.05, \mathrm{p}<0.001$ ); pen location on the right side (coef. est. $=0.08, \mathrm{p}=0.03$ ) and at the front of the experimental room (coef. est. $=$ $0.11, \mathrm{p}=0.003)$. By comparison, lower ear injury scores were associated with more enrichment (coef. est. $=-0.51, \mathrm{p}=0.005)$ and pen location at the front of the experimental room (coef. est. $=-0.4$, $\mathrm{p}=0.02$ ). These observed differences support the hypothesis that injuries to the body and ears arise from different risk factors. Although calculation of the minimum required sample size prior to conducting an experiment and selection of the inferential analysis method will contribute to the 
45 validity of the study results, conflict between the outcomes will require further investigation via 46 different methods such as sensitivity and specificity analysis.

47

48 Word count: 400

49

50 Key Words: Study design, sample size, mixed effect models, pig, animal health, animal welfare.

51 


\section{INTRODUCTION}

53 The statistician George Box stated "all models are wrong, but some are useful" (Box and Draper,

54 1987); which raises the question, how do we determine which statistical model, or in other

55

56

57

58

59

60

61

62

63

64

65

66

67

68

69

70

71

72

73

74

75 terminology, inferential analysis method, is most appropriate? In recent years, a spotlight has been directed at the transparency of animal research methodology, with low rates of methodological reporting being associated with less scientific rigour and lower reproducibility (Vogt et al 2016, Ionnides et al 2009, Kilkenny et al 2009). Articles pertaining to animal research have been criticised in the past for their design, statistical analysis and reporting (McCance, 1995; Kilkenny et al., 2009; Sargeant et al., 2010). The publication of a list of guidelines for animal research known as the ARRIVE guidelines (Kilkenny et al., 2010), has helped to improve the quality of animal research (Gulin et al., 2015). These guidelines highlight the importance of choosing the appropriate experimental assessments, sample sizes and statistical inferential analysis methods. It is important to ensure the sample size is sufficient to test the study hypothesis, but also bearing in mind the ethical and financial implications of using an unnecessarily large sample size within an experiment. There is a plethora of techniques to produce sample size estimates, and the appropriate technique will depend on the inferential analysis used for a study. Sample size can often be quite difficult to calculate for more complex designs, though the importance of conducting these calculations accurately has been well communicated, particularly in clinical trials literature (Freiman et al., 1978; Biau et al., 2008).

Discussion in this area naturally leads into consideration of the methodology of the statistical analysis conducted on the collected data. Many of the papers focussing on the quality of research using animals have primarily targeted experimental design, animal numbers, and reporting, but have not discussed the appropriate analysis of what can often be complex datasets. Precise replication of a published study is rarely performed, and typically different studies will use different experimental designs and statistical inferential techniques to address the question. Although this can make comparisons between published studies difficult, agreement in the overall conclusions under such circumstances can be considered strong evidence for the named association, though more subtle or complex relationships may potentially be missed. An identified significant treatment effect across 
studies through use of meta-analysis, is typically considered to be robust evidence for an association,

80 and also allows the magnitude of the effect size to be more precisely estimated than in single studies

81 considered in isolation (Borenstein et al., 2009). However meta-analysis also has limitations, for

82 example when few studies have been published in an area, when they differ substantially, or when the

83 inferential analysis used is inappropriate for the design.

84 Within the field of animal welfare, many published results on a particular issue are mixed or

85 conflicting, leading to somewhat mixed messages about what the most appropriate solution for an

86 identified welfare hazard might be. To some extent, it is possible that this is at least partly due to

87 publication bias (e.g. Hopewell et al., 2009; Brown et al., 2017) and the drive for novelty rather than

88 further support for a set of hypotheses in published research. However, the lack of agreement

89 between studies may be due to other factors - the differences may reflect genuine differences between

90 the studies, arising for reasons as yet unmeasured or unaccounted for. They may be due to the use of

91 indicators that have not been thoroughly validated in all respects for the species in question (Cronbach

$92 \&$ Meehl, 1955). Finally, the observed lack of agreement may be due to inappropriate statistical

93 analysis, leading to masking of true effects, or the discovery of false positives.

94 Even when two studies ask a very similar research question with largely similar methodology, mixed

95 results can emerge. A typical example of this can be found in studies that investigate causes, and

96 consequently solutions, for aggression in pigs. For example, Beattie et al. (1996) investigated whether

97 an enrichment object or floor space had more influence on pig behaviour. Their analysis showed that

98 duration of harmful behaviour was significantly higher in less enriched pens, and measured pig

99 aggressive behaviours had no significant association with space allowance. By comparison, Turner et

100 al. (2000) found that smaller space allowances were associated with more skin lesions and longer-

101 lasting aggressive events. These studies were similar in a number of respects, except that Turner et al.

102 (2000) regularly adjusted pen sizes to maintain a consistent stocking density (weight per $\mathrm{m}^{2}$ )

103 throughout the experiment, whereas Beattie et al. (1996) maintained pen dimensions (hence stocking

104 density would increase throughout the study). Consequently, the two studies are incomparable with

105 conventional meta-analytic approaches. Variation in the indicators used could also potentially explain 
differences in model outcomes For example, different indicators of injuries in pigs result in differences in the final conclusion, even if the studies use otherwise similar experimental designs and methods for inferential analysis. In relation to the provision of straw for pigs, different indicators of aggression have lead to different conclusions; for example, Lahrmann et al. (2015) found reduced shoulder injuries for straw-housed pigs, whereas Morgan et al. (1998) found that straw-housed pigs performed more aggressive interactions and Statham et al. (2011) and Arey and Franklin (1995) have both reported no significant effect of the provision of straw on outbreaks of aggression. Aggression can, and indeed, has been described and measured using a wide variety of indicators. Examples of indicators for aggression are: duration of fights and number of bites (Andersen et al. (2000); prevalence of giving/ receiving belly nosing, mounting, ear and tail biting, and biting the pen bars, chains or other pen details (Brunberg et al. (2011); the ratio of aggressive events to social interactions (Drickamer et al., 1999); skin lesions on different body areas (Desire et al., 2016). Frequently, there is little or no overlap between studies, or construct validation to demonstrate that all indicators recorded measure what they are proposed to measure (e.g. tail biting has been considered an indicator of aggression; however this has been reconsidered in more recent years, e.g. Taylor et al., 2010).

Here we used a study investigating aggression in pigs to compare differences between two areas for the assessment of skin injuries (believed to be indicative of aggression in pigs), an ear score and a composite body score (Conte et al. 2012), and the effects of analysing the data via four inferential methods: (i) generalised linear models; (ii) repeated measures analysis; (iii) linear mixed effect models; and (iv) linear mixed effect models for repeated measures. We compare the significant associations between the two injury assessments and the covariates detected via the exploratory and four methods of inferential analysis. These four approaches were chosen because, to varying degrees, these models could account for some of the features of the data and model parameters could be directly interpreted.

Methods (i)-(iii) were considered sub-optimal relative to (iv), as these models were unable to account for correlation in the repeated measures, and /or random effects from the hierarchical structure in the data (pens within replication). We hypothesised that not accounting for random effects from the pens 
within replication and correlation between repeated measures will either result in additional spurious

134 relationships and/or mask possible significant relationships between our injury assessments and the

135 covariates. By ignoring random effects, we hypothesise there will be more statistically significant

136 associations with environmental factors, and by ignoring the repeated measurements, we hypothesise

137 the association between injury score and time covariate will be more complex.

138 We investigated the effects of sample size within multilevel designs by analysing the data from

139 different replications ( $\mathrm{n}=18$ pigs $* 4$ pens per replicate) as separate studies, and comparing the

140 coefficient estimates from each of these analyses. A reduced sample size leads to a decrease in power,

141 which means it is more difficult to identify the environmental factors associated with the injury scores.

142 We hypothesize, that with a reduced sample size, there will be fewer statistically significant

143 associations between injury scores and environmental factors.

\section{2. METHODS AND MATERIALS}

\subsection{Animals and Housing}

147 The study was conducted at the Agri-Food and Biosciences Institute, Hillsborough, County Down,

148 Northern Ireland. The study used commercial crossbreed PIC 337 (Large White x Landrace) pigs.

149 Pigs received a commercial weaner diet ad libitum and water was always available, according to the 150 standard practices on the farm.

151 Each pig was weighed when they were four weeks and again at ten weeks old. The pigs' sex and weights at 4 weeks of age were used by the stockman to balance the groups to achieve a similar average weight and 50:50 sex ratio in each group of 18 individuals. Groups were then allocated at

154 random to one of four pens. The pigs remained in these pens for a period of approximately six weeks, and the study was replicated twelve times, which led to a sample size of 864 . 
Pigs were assigned to one of four pens for the study that were contained within an experimental room

157 situated in a long shed, which was divided into a series of similar rooms, with floor to ceiling solid

158 walls between each room. Two types of pen environment were used within this study. Pens 1 and 3

159 were classed as more enriched environments; these pens were $2.18 \mathrm{~m} \times 5.16 \mathrm{~m}$ in dimension with

160 deep straw bedding (replenished weekly). Pens 2 and 4 were classed as less enriched environments,

161 these were $2.18 \mathrm{~m} \times 3.42 \mathrm{~m}$ in dimension, and no straw was provided. Both pens had floors constructed from concrete and were partially slatted, however in the more enriched pens (1 and 3) the slats were covered with plywood to prevent straw falling into the slurry system. In all pens, suspended wooden blocks were provided as standard enrichment.

Pens 1 and 2 were located on the left side of the experimental room and pens 3 and 4 were located on the right. The adjacent room on the right (next to pens 3 and 4) almost always contained weaner pigs, whereas the adjacent room on the left (next to pens 1 and 2) was frequently empty, or was occasionally used to house sows that could not enter farrowing crates. The difference in directional noise from each adjacent room was balanced in the experimental design by having one pen of each treatment type on both sides of the room. Two of the four pens were located next to the front of the room (pen 2 and pen 3), and the other two pens were located at the back next to an internal corridor.

172 The pigs were kept commercially, hence decisions relating to culling and health were made by the farm manager, as part of the standard on-farm procedures. Outbreaks of aggression leading to injury were observed only on video footage, analysed typically several weeks after recording took place.

175 Animals that were observed to have high body scores were reported to farm staff, and monitored 176 closely by farm staff and researchers for a period of 7 days after. No animals were culled for the 177 purposes of this study, though as noted in section 2.3 , a small number of animals ( $\mathrm{n}=9$ out of 862 pigs) died during the study period due to poor health or failure to thrive.

\subsection{Assessment of Injury}

An assessment of each individual's injuries was completed at three time points after entering the pens: 
scored on the following body areas: left and right ear; snout; left and right shoulder; front and back legs; left and right flank; left and right hindquarter; and back; using a six point scaling system, as defined in figure 1 (Conte et al. 2012). As part of the standard practice on the farm, $50 \%$ of the tail was docked within the first 24 hours after birth for every pig, this meant that tail score had limited value as an indicator for aggression.

\subsubsection{Indicators of Aggression}

Ear and body score were considered as indicators of aggression. At each assessment time point, the ear score was recorded as the higher observed injury score on either the left or right ear (possible score $0-5$ ), and the body score was recorded as the sum score of the back, left and right shoulder, flank and hindquarters scores (possible score $0-25$ ).

Due to the method used to construct the body score, based on the Conte et al (2012) scale, the two elements of frequency of injury and severity are confounded, especially for lower values. In our dataset, body score ranged between zero and 25 , suggesting body score could be analysed as a continuous variable. A histogram plot of the log transformed body score implied we could assume the data followed a Gaussian distribution.

Each ear was scored on a scale between zero and five, with a score of zero signifying no injuries or damage, and a score of five indicating the presence of many deep red lesions. As very few pigs were identified with a score of 3 or more, categories 3 to 5 were combined, so that the ear score categories represented: $0=$ no injuries; $1=$ one small superficial lesion; $2=$ more than one small, superficial lesion; or one red (ie deeper than score 1) superficial lesion; 3 = one or more deep lesions, or more than one red superficial lesions. Initial exploratory analysis suggested that the relationship between the housing conditions, sex and weight were similar for pigs with an ear score of 0 or 1 . Therefore, these two groups were combined to simplify subsequent inferential analyses.

\subsection{Statistical Analysis}


207 As injury assessments were made at three irregularly spaced points in time, the assessments for an

208 individual pig could be correlated, but the strength of the correlation may differ because of the

209 variable time differences. Replicating the study 12 times may cause significant random effects for

210 each pen within replication. The differences could be caused by the combination of pigs within a pen,

211 or even associated with unmeasured external influences (e.g. weather conditions, handler behaviour,

212 noise). Using weight at 4 and 10 weeks of age, we produced estimates of each individual's

213 intermediate weights by fitting a linear model between the two time points. Although growth is

214 usually statistically modelled by a curve, plots of the expected growth curves in Carr (1998) indicated

215 that a linear estimate of pig weight would be an appropriate approximation over the short time scale

216 used in this study.

217 We calculated individual relative weights in each pen within replication, in line with previous

218 research indicating that an individual's relative size compared with its group mates is more important

219 than its actual size (Nettle et al., 2013). Andersen et al. (2000) found no significant difference in

220 number of bites between groups of pigs with low and high weight variability, which suggested

221 removing pen differences would have no adverse effects. This is similar to comparing a pig's weight

222 rank, but also accounts for variable weight differences between pigs.

223 Missing data were due to human error in data entry, and death or culling of the individual pig during the course of the study, either due to poor health or failure to thrive.

225 The plots and statistical analyses were produced using the statistical program R (Team, 2015) using

226 the multgee (Touloumis, 2016), ordinal (Christensen, 2015), and lme4 (Bates et al., 2015) packages to

227 produce the statistical models.

229 Before applying any statistical test or fitting a statistical model to data, it is important to perform appropriate exploratory analysis. Choosing the right method to explore the data will depend on the 
question being addressed. As these data consisted of observations measured over time, we aimed to

232 explore how body and ear score changed over time.

233 We plotted each pig's body score over time and fitted a Gaussian kernel smooth estimator to pigs

234 within each category (i.e. by treatment enrichment level). A kernel estimator is a non-parametric method of fitting a line between two continuous variables. If there is uncertainty about the form of this relationship (i.e. linear, quadratic, etc.), visual inspection of plots of the data can provide insight

237 into this. An appropriate bandwidth is determined, with bigger bandwidths creating smoother lines.

238 We selected a bandwidth of 15 , as injury assessments took place every 14 days on average (more 239 details of kernel estimators can be found in Wand and Jones (1994)). As we were treating ear score as

240 an ordinal variable, we looked at the proportional change of pigs within each category, and used the

241 same methods as outlined above for body score.

\subsubsection{Inferential Analysis}

243 The data from this experiment possessed a hierarchical structure, where we had repeated

244 measurements for each pig, within a pen, within a replication. There are various methods that can be

245 applied to this type of data, depending on the assumptions one makes. We compared the results of

246 four methods of analysis on body and ear score, where each method considered different aspects of

247 the study design: (i) ignored the study design; (ii) considered correlation in the repeated

248 measurements; (iii) considered random effects from the hierarchical structure; (iv) considered the

249 correlation structure and the random effects. Table 1 provides a comparison of the different inferential

250 methods considered in this paper. Depending on the study design, it indicates which inferential

251 method would be appropriate for different types of data.

(i) Ignoring study design (without accounting for repeated measures or hierarchical structure)

253 To demonstrate the effects of ignoring the study design completely, i.e. not accounting for repeated

254 measures of individuals and random effects, we fitted a generalised linear model (GLM) to body and 
ear score. Specifically a log linear model (LLM) was fitted to body score and a cumulative logistic regression model (CLM) was fitted to ear score.

258 As we assumed body score is continuous, we performed a multivariate analysis of covariance

259 (MANCOVA) with a Gaussian distribution. This methodology compares the means of all the different

260 possible groups and determines whether a significant difference is present when accounting for a

261 possible time-dependent correlation between the assessments. We accounted for the replications

262 within this inferential analysis using an error structure for individuals within replications.

263 MANCOVA assumes that the assessments measured are taken at equally spaced points in time, and

264 the difference in time is the same for each individual. Only individuals with complete data are

265 included.

266 As ear score is an ordinal variable, we fitted a cumulative logistic regression model for repeated

267 measures. To account for repeated measurements of the ear score, the parameters were estimated via generalized estimating equations (GEE), which allow for the presence of a possible time-dependent correlation between ear score assessments made at different times. However, a covariate for the replication was also included to account for the possible differences between replications.

272 To remove the effect of the repeated measures we produced a summary variable for each pig. The summary variable for body score was simply the mean of the log transformed body score across each

274 of the three repeated measures. The summary variable for ear score was slightly more complicated.

275 Often categorical variables are summarised by their median or modal value. However, as the median 276 and mode are not influenced by extreme values, it meant that severe injuries were missed. Therefore, 277 we summed the ear score for each replication, then combined some of the categories according to the 278 frequency and level of injury the category represented to bring the score in line with the original scoring system. The new ear score categories were $0=$ less than 2 occurrences of superficial lesions, 
or 1 occurrence of a deep lesion; $1=1$ occurrence of a deep lesion and 1 occurrence of a superficial

281 lesion or 3 occurrences of superficial lesion; 2 more than 1 occurrence of a deep lesion.

To account for the random effects of pen within replication we fitted a mixed effects linear regression model (LME) to the mean log body score

$$
y_{i, j}=\alpha+X_{i, j} \boldsymbol{\beta}+Z_{i, j} \boldsymbol{\delta}_{i}
$$
score

$$
\operatorname{logit}\left(\operatorname{Pr}\left[Y_{i, j}<k\right]\right)=\alpha_{k}+X_{i, j} \boldsymbol{\beta}+Z_{i, j} \boldsymbol{\delta}_{i}
$$

where: $y_{i, j}$ is the mean log body score; $Y_{i, j}$ is the ear score category for $k=0,1,2 ; \alpha$ is the intercept whereas $\alpha_{k}$ is the intercept for the $k^{\text {th }}$ cumulative logit; $\boldsymbol{\beta}$ is a vector of fixed effects coefficient estimates; $X_{i, j}$ are the fixed covariates design vector for the $j^{\text {th }}$ pig, in the $i^{\text {th }}$ replication $\boldsymbol{\delta}_{i}$ is a vector of the random effects for replication $i$; and $Z_{i, j}$ is a design vector of the random effects.

An important difference between the GLM and a mixed effects model comes from the estimation of

294 the variance. In a GLM only the variance of the individual pigs is required, whereas now an estimate

295 for the variance for the individual pigs and the replications is required.

To account for both the hierarchical design and repeated measurements within this study, we fitted the log linear and cumulative logistic, mixed effects model as defined in eEquation 3Equation 4:

$$
\log \left(y_{i, j, t}\right)=\alpha+X_{i, j, t} \boldsymbol{\beta}+Z_{i, j, t} \boldsymbol{\delta}_{i},
$$




$$
\operatorname{logit}\left(\operatorname{Pr}\left[Y_{i, j, t}<k\right]\right)=\alpha_{k}+X_{i, j, t} \boldsymbol{\beta}+Z_{i, j, t} \boldsymbol{\delta}_{i}
$$

303 These are very similar to Equation 1Equation 2, and in fact, the mathematical representation only 304 requires the addition of a subscript $t$ to denote the time element in the random effects model. See 305 Twisk (2012) for more details on this type of analysis.

306 Computationally, as we are treating body score as a continuous Gaussian distributed variable, 307 estimation of the coefficients and the variance for the replications and individuals in Equation 3 can 308 be accomplished via GEE. However, there is no software available currently which can produce a mixed effects cumulative logistic regression model with repeated measures where the correlation between each observation depends on the time difference between repeated measures.). We concluded that as we only had three repeated observations, estimation of the random effects was more important

312 than using GEE to account for a time dependent correlation structure for ear score. However, a

313 random effect term for each pig was included instead, as it assumes the correlation between 314 observations is constant over time.

316 To investigate the effects of small sample sizes, a repeated measures model was fitted to the data of 317 each replication. This led to 12 statistical models, one for each replication, which each consisted of 72 318 pigs per model/replication (18 pigs assigned to 1 of 4 pens), with a maximum of three skin lesion assessments each, giving a total of number of observations of 216 per model. Each GLM consisted of the same covariates, which were equivalent to the covariates in the final hierarchical repeated measures model.

\section{RESULTS}


For 862 individual pigs we had a measurement for at least one of the injury assessments. For body score there were two pigs with missing data for the first observation, seven pigs with missing data for the second observation and nine pigs with missing data for the third observation. For ear score there were three pigs with missing data for the first observation, seven pigs with missing data for the second observation and 10 pigs with missing data for the third observation.

\subsection{Body Score}

330

\subsubsection{Exploratory Analysis}

331 The plots of the kernel smooth estimators in figure 2 a) - e) depict a cubic relationship with time. The kernel estimators of log body score are between 1 and 2 at the first examination (day 0), with a decline in $\log$ body score by the second examination (days 8-17), but by the third examination (days 29-39) there is an increase. All covariate groups mirror this pattern.

However, the slopes for each replication varied, as shown in figure 2 a), thus implying a random slope for replication over time was required. Figure 2 b) of the Gaussian kernel smooth estimators for each pen was used to determine whether different housing features were worth investigating. It is clear that pigs within pen 3 tended to have a higher body score than any of the other three pens, which all appeared to be quite similar. There was a difference between the intercept and a slight difference

340 between the slopes for each pen.

341 The plots in figure $2 \mathrm{c}$ ) to e) further identify differences between the pens. Comparing the score of the different environments in figure $2 \mathrm{c}$ ), the difference between the less and more enriched environments is only evident after approximately 14 days. This implies an interaction between time and environment. The plot in figure $2 \mathrm{~d}$ ) shows that pigs in the pens to the front of the experimental room

345 had a consistently higher body score than pigs in the pens located at the back. We also observed that

346 pigs in pens on the right side of the room had a higher body score than those in pens on the left side of 347 the room, as shown in figure $2 \mathrm{e}$ ). 
348 The plot in figure $2 \mathrm{f}$ ) is a scatter plot of body score by standardised relative weight. The blue line is

349 the kernel smooth estimator using a bandwidth of 0.75 . Less than $3 \%$ of the standardised weight

350 values were either $>2$ or $<-2$, which meant there were insufficient values to produce a reliable

351 estimate of the relationship between body score and relative weight. However, the plot suggested that

352 for a relative weight between -2 and 2, the relationship was linear and as weight increased so did log

353 body score.

\section{$354 \quad 3.1 .2$ Inferential Analysis}

355 Table 2 contains all the summary statistics for the fixed effects (coefficient estimate, standard error,

356 Student's t-value and p-value) for the most appropriate model, (iv) LLME + GEE, and the p-values

357 for all fixed effects for the three comparison methods, (i) LLM, (ii) MANCOVA and (iii) LLME. If a

$358 \mathrm{p}$-value was greater than 0.05 it was not included in the table. In all the statistical models the

359 enrichment level, location of the pen (left/right side, front/back of the experimental room) was

360 significantly associated with body score. Relative weight was a significant component in 3 out of the

3614 statistical models.

362 The LLME + GEE model accounted for a random intercept and slopes over time for pens within 363 replications, and a Gaussian correlation structure between observations for each pig. There was a 364 significant cubic relationship with time, this can also be seen in figure 2 (a)-(e) of the kernel

365 estimators. The significant relative weight coefficient implied that a unit increase in relative weight

366 resulted in a 0.05 increase in log body score, which equates to a 5\% increase in body score. On

367 average, pigs on the right side of the room had a 0.094 higher log body score, i.e. their body score was

$3689.9 \%$ higher than those on the left side of the room. Also pigs with more enrichment and those in pens

369 located at the front of the experimental room had higher log body scores by $0.124(13.2 \%$ increase in

370 body score) and 0.09 (9.4\% increase in body score), respectively. 
372 Figure 3 a) is a box plot of the coefficient estimate when using GEE to analyse each replication; when

373 the random effect for replication was not included, with the fixed effect coefficient estimates under

374 LLME + GEE model (table 2) included as a red cross. The box plot for relative weight was the only

375 one where the whiskers of the plot did not include zero, implying this was the only covariate with a

376 significant association with $\log$ body score for all but one replicate. This suggested that the coefficient

377 estimate for relative weight should remain fairly consistent across replications. For pen location (left/

378 right, front/back of the experimental room), and more enriched pens, the coefficient estimates showed

379 greater variance.

380 The median coefficient estimates were: weight $=0.04$; right side of experimental room $=0.1$; location

381 to the front $=0.14$; and more enriched environment $=0.11$. Comparing these values with the

382 coefficients estimates of the LLME + GEE model in table 2 we see that these values are quite similar,

383 and encouraging as a form of sensitivity analysis. Within one replication, there are 216 observations.

384 If we were to perform a t-test on these 216 observations to detect the largest effect size of 0.14 in $\log$

385 body score, assuming the standard deviation was 0.6 (estimated from the entire dataset), then we

386 would have $\approx 40 \%$ power to detect this difference. This does not account for the repeated measures,

387 which would reduce the power further.

\subsection{Ear Score}

\subsubsection{Exploratory Analysis}

390 From figure 4 there is evidence of a cubic relationship between ear score and time when comparing

391 the proportion of pigs with an ear score of 0 with 1 and/or 2 (all plots on the left), where there is a

392 decrease, plateau, then further decrease. However, the plots comparing the proportions observed in 0

393 and/or 1 with 2 (plots on the right) appear to be exponentially decaying.

394 The plots in figure 4 show the proportional change in the pigs observed within each ear score group

395 with Gaussian kernel estimators to convey how the relationship between ear score changes over time

396 for different housing features. In figure 4 a) the variability in the shape of the relationship between ear 
397

398

399

400

401

402

403

404

405

406

407

408

409

410

411

412

413

414

415

416

417

418

419

420

421

score and time for the different replications indicate a different slope for each replication over time is required. However, in figure 4 b) the estimators for each pen have a similar shape, but different intercepts. There are clear differences in figures $4 \mathrm{c}$ ) and d) between environment and location next to the front or the back of the experimental room.

\subsubsection{Inferential Analysis}

Table 3 shows all the summary statistics for fixed effects (coefficient estimate, standard error, Student's t-value and p-value) for the cumulative logistic mixed effects regression model with random effect for pigs, (iv) CLME +1 , and significant $p$-values for fixed effects from the three comparator methods (i) CLM, (ii) GEE and (iii) CLME. Within each statistical model, ear score was shown to have a significant association with the level of enrichment and the front/back pen location.

The CLME+1 model included random intercept and slope terms for pen within replication to account for the differences between replications over time, and a random intercept for each pig to account for the correlation between repeated measures. To discuss our findings, we use odds ratios (i.e. exponential transformation of the coefficients), so we can quantify the percentage increase or decrease in odds that will result in the increase or decrease in ear injury score. In the CLME +1 model, pigs in more enriched pens had 40\% lower odds (Confidence Interval, CI: $14 \%, 58 \%$ ) of having a higher ear score compared to pigs in less enriched pens. Similarly, pigs in a pen located at the front of the room had $33 \%$ lower odds (CI: 5\%, 53\%) of having a higher ear score.

\subsubsection{Small sample sizes}

We fitted a CLME model to each replication with a random intercept for each individual. Figure $3 \mathrm{~b}$ ) contains the box plot of the coefficient estimates from the ordinal logistic regression of ear score for each replication. The fixed effect coefficient estimates under CLME+1 (table 3) are included as a red cross in figure $3 \mathrm{~b}$ ). There was a wide range of values for the coefficients from each replication (median coefficient estimate for more enriched environment $=-0.55$; front of experimental room $=$ 0.21). Comparing the coefficient estimates for CLME and CLME+1, there was little difference 
between pen enrichment estimates (0.04), but a larger difference between pen location estimates

423 (0.19).

\subsection{Inference method comparisons}

425 For both types of injury score, the key associations between the injury score and environmental 426 factors were statistically significant across all four statistical models. Although, the magnitude of the 427 relationship and the direction was not always the same between the most appropriate statistical model from approach (iv), and the other three statistical models, using methods (i) to (iii). The model via approach (iii) for both injury scores provided no insight into changes in injury over time, as this information was removed when summarising the injury scores.

431 Table 2 details the level of association between body score and the environmental factors for each 432 inferential method. Approach (i), the LLM, did not account for the repeated measure correlation or random effects, and there was an additional significant association between body score and tail injury. Whereas for approach (ii), the MANCOVA, which only accounted for repeated measurements, there was a significant association between body score and sex. Neither of these associations were evident in the exploratory analysis or in the most appropriate approach (iv). However, the association between

437 body score and weight was not statistically significant in approach (iii), the LLME model, but the 438 evidence from exploratory analysis and most appropriate model indicated there was a relationship 439 between these two variables.

440 In table 3 the statistical models from methods (i), CLM, and (ii), GEE, did not account for the random

441 effects of pen within replication that led to high order degree polynomials with the day, 7 and 5

442 respectively. There was no evidence in the exploratory analysis or the final most appropriate model

443 (CLME + 1), that this type of association between ear score and time was valid. 
446 Comparing models where each incorporated different aspects of the study design demonstrated how

447 important using the most appropriate inferential analysis is when producing valid results. By

448 appropriately accounting for all sources of variation within the multilevel structure of the data (i.e.

449 pens within replications) and considering the potential time-dependent correlation between

450 observations, we increased the likelihood of identifying the true associations between the covariates

451 and injury scores. We also found that there was a strong agreement between exploratory and

452 inferential analysis, and associations seemed to be plausible.

453 In the most appropriate model for the data (repeated measures, mixed model), the strong significant

454 association of ear and body injury score with the non-linear time component is suggestive of a

455 complex relationship between behaviour and time. This observation was only possible because of the

456 repeated observations within pigs, and further validated by the replications of the study. Although the

457 variation in the inter-assessment interval time increased the statistical difficulty of the analysis, it did

458 mean that there was more information available about changes in injury score over a wider range of

459 interval differences. Ear and body injury score were both associated with the enrichment level and

460 front location of pen within the experimental room, although the direction of this association changed

461 for both covariates between injury scores. More enriched pens (coef. est. $=-0.51, p=0.005$ ) and pens

462 at the front of the experimental room (coef. est. $=-0.4, p=0.02)$ were both associated with a

463 reduction in ear score, whereas those in more enriched pens (coef. est. $=0.09, p=0.02$ ), and pens at

464 the front of the experimental room (coef. est. $=0.11, p=0.003)$ had a higher body score. Body score

465 was also associated with weight and pen location on the right side of the experimental room, such that

466 as weight increased so did body score (coef. est. $=0.05, \mathrm{p}<0.001$ ), and those pigs in pens on the right

467 side of the experimental room also had a higher body score (coef. est. $=0.08, p=0.03$ ).

468 In this study, we investigated the impact of fitting statistical models that account for none, some and

469 all of the known structural features of a multilevel dataset. We also analysed the effect of small

470 sample size upon the most appropriate model. Similar investigations comparing inferential analyses

471 have been conducted in human and non-human medical literature (Hu et al., 1998; Wang and

472 Goonewardene, 2004), though this is the first example to the authors' knowledge in animal welfare. 
473 In using an analytical approach that did not match the study design (approach (i): CLM), variance

474 within the dataset that was associated with either the hierarchical structure or the correlational

475 structure between repeated observations was not accounted for. This approach (CLM) led to

476 predictions of a complicated relationship between ear injury score and time, with a 7-degree

477 polynomial predicted to describe the relationship. For body score, the CLM predicted a cubic (i.e. 3-

478 degree polynomial) relationship with time, just as was predicted by the most appropriate model

479 (CLME+1). The high degree polynomial relationships predicted here result from poor estimation of

480 variance, due to the models attempting to explain variation in the data using only the covariates,

481 without the underlying hierarchical structure accounted for.

482

483

484

485

486

487

488

489

490

491

492

493

494

495

496

497

Including the correlation of the repeated measurements for approach (ii) via MANCOVA for body score and GEE for ear score did increase the p-values, but it did not account for the substantial variation caused by the random effects. Hence, there was an additional relationship between body score and sex, and the association between ear score and day was now a 5-degree polynomial. One substantial drawback back with MANCOVA is the strict format required of the data, i.e. equally spaced repeated measures with no missing values. Using GEE analysis is more flexible and the observations do not necessarily have to be equally spaced. However as the correlation coefficients between repeated measurements of ear score were all less than 0.3 , and the differences between the estimators for replications and pens from the plots in figure 3 a) and b) appeared quite high, this suggested the random effects terms for replication and pen were more important than accounting for the correlation structure between repeated measurements. By replicating the study, we were able to gain insight into differences between pens, which we had not considered for inclusion in our experimental design prior to conducting the study; in particular, this would have been beneficial for the location of the pens within the experimental room. Although we accounted for differences in noise level with left/right side counter-balancing of the treatments, and accounted for potential differences between pens at the front (near the door) versus at the back of the room with front/back counter-balancing of treatments, we did not rotate the pens, which would have allowed us to account for the additional locational differences detected in the data. Although we were unable to fully explain 
the reason for differences between pen locations within the experimental room, we were able to

501 identify that pen location was a source of variation and we could therefore statistically remove any

502 undue influence this was having on other covariates within the model. Differences observed between

503 replications could be related to weather conditions, handlers and many other features not measured as

504 part of this study. Despite being unable to quantify all variation between replications, we believe that replication on other farm sites would help to build up a more general picture across contexts.

506 Summary measures of both body and ear score were used in approach (iii), which resulted in lost

507 information about the nature of the relationships of body and ear score across time. Using this

508 approach, we were unable to identify a significant association between body score and weight via the

509 LLME model, but we detected a significant relationship between ear score and weight using the

510 CLME, as compared to the final appropriate model.

511 In the final approach (iv) for body score and ear score, there was evidence of a cubic relationship with

512 time for both injury scores. However, the direction of the coefficient estimates for day, day ${ }^{2}$ and day ${ }^{3}$

513 differed between body and ear injury scores. For body scores, the coefficients for time were positive

514 for day and day ${ }^{2}$ and negative for day ${ }^{3}$, whereas for ear score they were negative for day and day ${ }^{3}$ and

515 positive for $\mathrm{day}^{2}$. This result implies that the underlying behaviour indicated by proxy from these

516 injury scores changed over time. For example, the initial decline in scores could be associated with

517 pigs becoming acquainted with one another as a hierarchy within a pen was established within the

518 first week (Barnett et al., 1994; Arey, 1999).

519 In both the final ear score and body score statistical models there was a significant association with 520 pen location (front/back of the room) and enrichment level (see section 3.2.2). Pigs in pens located at 521 the front of the room had lower odds of having a higher ear score (table 3), but higher odds of a higher 522 body score (table 2). Pigs in more enriched pens had lower ear scores (as described in section 3.2.2,

523 table 3). This result supports previous findings that aggressive events are reduced in larger pen sizes

524 (Fraser et al., 1991; Turner et al., 2000). Whereas the LME + GEE model for body score implies that 525 more enriched pens resulted in higher body injury scores. 
Finding clear differences in the predictors for ear and body scores lends support to the hypothesis that

527 they have different underlying causes. Injuries to the ear are mainly received during aggressive

528 interactions (McGlone, 1985). Injuries to the body on the other hand, whilst accrued through

529 aggression, can also be the result of increased activity and play (Munsterhjelm et al. 2009; Camerlink

530 et al., 2013). Unfortunately, as tails were docked at birth we were not able to use tail injury as another

531 comparator, although research suggests that the majority of tail injuries reflect exploratory motivation

532 rather than aggression (Taylor et al., 2010). Applying a similar study to undocked pigs may provide

533 further detailed insight into aggression and the underlying motivating behaviours that lead to injuries.

534 Statistical techniques used to determine the validity in medical screening tests, such as a receiver

535 operator curve (ROC) analysis (Fawcett, 2006) or Bland-Altman test (Bland \& Altman, 1986), may be

536 used to compare indicators of aggression to determine if they are a measure of the same quantity.

537 Whilst the final model selected is appropriate for the experimental design, it is not perfect. There are currently no developed statistical methods available to analyse categorical outcome variables with a

539 time dependent correlation structure between repeated measures within a hierarchical model (such as

540 the random effects of replications within pens described within section 2.1). As such, we could not

541 account for both the correlational structure and hierarchy of the study design within current statistical

542 methodology. One possible solution could be to develop a statistical model with a probit link rather

543 than a logit link, as the probit link is associated with the Gaussian distribution, and it may be easier to

544 define a time dependent correlation structure with this compared to the logit link. However, the

545 interpretation of the probit link can be difficult as there are no direct interpretations of the coefficients,

546 instead it is necessary to refer to the marginal effects of the regressors (see Liao (1994) for more

547 details), and the estimation of the coefficients would be computationally intensive.

548 Differences between the results of the four inferential methods highlight the importance of initial 549 exploratory analysis in determining whether resulting significant associations are realistic, particularly

550 as all four methods used are technically appropriate, albeit with varying degrees of fit to the

551 experimental design. Strong evidence of a relationship in the exploratory analysis should translate to a

552 significant association observed within the inferential analysis. Although measures were taken into 
account for layout of the experimental room, it was not possible to completely account for the extent

554 of this effect, and it was through exploratory analysis that we were provided with greater insight into

555 the magnitude and nature of the effect.

556 By analysing each replication separately, we were able to demonstrate how sample size affects the

557 final coefficient estimates. The decrease in data resulted in insufficient power to detect significant

558 associations, although the calculated medians of almost all the replications' coefficient estimates were

559 consistent with our full final models. The results clearly demonstrate that analysis of small sample

560 sizes may lead investigators to believe there was no association between the indicators for aggression

561 and covariates, whereas it could be the study is under-powered to detect the effect size (i.e. the

562 conclusion would be a type 2 error). As a simple demonstration, we performed a power calculation to

563 detect a mean difference in body score of 0.18 and standard deviation of 0.6 , based on summary

564

statistics of enrichment level in the fifth week. The power calculation found that to detect such a

565

difference with $80 \%$ power at the $5 \%$ level of significance, a sample size of 176 pigs (total 352 )

566 assigned to each enrichment level was required.

567

This study demonstrates through examples, how the type of indicator measured, the sample size and choice of statistical analysis can affect model outputs and conclusions drawn. We also highlight the importance of using an appropriate indicator to reflect the behaviour under investigation. The correct inferential analysis is important for meaningful results, which are not only plausible, but also supported by the exploratory analysis. To ensure the quality of animal science reports it is vital that a

572 study consists of an appropriate sample size, with statistical analysis appropriate for the study design.

573 These findings provide further support for the ARRIVE guidelines, but we feel that additional steps

574 may improve the quality of research by ensuring studies are designed based upon the inferential analysis best equipped to answer the research question. It may be valuable to consider following similar procedures as in medical trials with the formulation of a protocol and detailed documentation

577 of any unexpected and additionally planned deviations, which may subsequently affect the inferential

578 analysis. This way, while best laid plans may still go awry in practice, there will be a clear plan to

579 ensure that robust and appropriate analysis of the data can still be conducted. 
581 The authors would like to thank AFBI for use of experimental room and care of the animals.

\section{ETHICS STATEMENT}

583

All procedures described were approved by the University of Lincoln's Ethics Committee on 8/9/2015,

584 code COSREC62. This research was conducted at the Agri-Food and Biosciences Institute, Northern Ireland and conformed to the Association for the Study of Animal Behaviour's guidelines on the use of animals in research: http://asab.nottingham.ac.uk/ethics/guidelines.php.

\section{FUNDING STATEMENT}

588 This work was supported by the BBSRC (grants BB/K002554/1 and BB/K002554/2). MF was 589 supported by a Department for Employment and Learning Northern Ireland studentship and Queen's 590 University Belfast.

\section{REFERENCES}

592

Andersen, I.L., Andenæs, H., Bøe, K.E., Jensen, P., Bakken, M., 2000. The effects of weight asymmetry and resource distribution on aggression in groups of unacquainted pigs. Appl. Anim. Behav. Sci. 68, 107-120.

595

Arey, D.S., 1999. Time course for the formation and disruption of social organisation in group-housed sows. Appl. Anim. Behav. Sci. 62, 199-207.

597

Arey, D.S., Franklin, M.F., 1995. Effects of straw and unfamiliarity on fighting between newly mixed growing pigs. Appl. Anim. Behav. Sci. 45, 23-30.

Barnett, J.L., Cronin, G.M., McCallum, T.H., Newman, E.A., 1994. Effects of food and time of day on aggression when grouping unfamiliar adult pigs. Appl. Anim. Behav. Sci. 39, 339-347.

Bates, D., Maechler, M., Bolker, B., Walker, S., 2015. lme4: Linear mixed-effects models using Eigen and S4, http://CRAN.R-project.org/package=lme4. 
603

604

605

606

607

608

609

610

611

612

613

614

615

616

617

618

619

620

621

622

623

624

625

626

627

628

Beattie, V.E., Walker, N., Sneddon, I.A., 1996. An investigation of the effect of environmental enrichment and space allowance on the behaviour and production of growing pigs. Appl. Anim. Behav. Sci. 48, 151-158.

Biau, D.J., Kernéis, S., Porcher, R., 2008. Statistics in Brief: The Importance of Sample Size in the Planning and Interpretation of Medical Research. Clinical Orthopaedics and Related Research 466, $2282-2288$

Bland, J. M. \& Altman, D. G. (1986). Statistical methods for assessing agreement between two methods of clinical measurement. Lancet i, 301-310.

Borenstein, M., Hedges, L. V., Higgins, J. P. T., \& Rothstein, H. R. (2009). Introduction to metaanalysis. Chichester: Wiley.

Box, G.E., Draper, N.R., 1987. Empirical model-building and response surfaces. Wiley New York. Brown, A.W., Mehta, T.S., Allison, D.B., 2017. Publication bias in science: What is it, why is it problematic, and how can it be addressed? In: The Oxford Handbook of the Science of Science Communication. Ed: K. Hall Jamieson, D. Kahan, D.A. Scheufele. Oxford University Press, New York, USA.

Brunberg, E., Wallenbeck, A., Keeling, L.J., 2011. Tail biting in fattening pigs: Associations between frequency of tail biting and other abnormal behaviours. Appl. Anim. Behav. Sci. 133, 18-25.

Camerlink, I., Turner, S. P., Bijma, P., Bolhuis, J. E. (2013). Indirect genetic effects and housing conditions in relation to aggressive behaviour in pigs. PloS one.;8:e65136.

Carr, J., 1998. Garth Pig Stockmanship Standards. 5m Publishing.

Christensen, R.H.B., 2015. ordinal: Regression Models for Ordinal Data, http://www.cran.rproject.org/package $=$ ordinal.

Conte, S., P. G. Lawlor, N. O’Connell, and L. A. Boyle. 2012. Effect of split marketing on the welfare, performance, and carcass traits of finishing pigs1. J. Anim. Sci. 90:373-380.

Cronbach, L. J. \& Meehl, P. E. (1955). Construct validity in psychological tests. Pyschological Buletin. 52: 281-302. 
Desire, S., Turner, S.P., D'Eath, R.B., Doeschl-Wilson, A.B., Lewis, C.R.G., Roehe, R., 2016. Prediction of reduction in aggressive behaviour of growing pigs using skin lesion traits as selection criteria. Animal 10, 1243-1253.

Drickamer, L.C., Arthur, R.D., Rosenthal, T.L., 1999. Predictors of social dominance and aggression in gilts. Appl. Anim. Behav. Sci. 63, 121-129.

Fawcett, T. (2006). An introduction to ROC analysis. Pattern Recognition Letters 27: 861-874.

Fraser, D., Phillips, P.A., Thompson, B.K., Tennessen, T., 1991. Effect of straw on the behaviour of growing pigs. Appl Anim Behav Sci 30, 307-318.

Freiman , J.A., Chalmers , T.C., Smith , H.J., Kuebler, R.R., 1978. The Importance of Beta, the Type II Error and Sample Size in the Design and Interpretation of the Randomized Control Trial. New England Journal of Medicine 299, 690-694.

Gulin, J., Rocco, D., Garca-Bournissen, F., 2015. Quality of Reporting and Adherence to ARRIVE Guidelines in Animal Studies for Chagas Disease Preclinical Drug Research: A Systematic Review. PLoS Negl. Trop. Dis. 9, 1-17.

Hopewell, S., Loudon, K., Clarke, M. J., Oxman, A. D., Dickersin, K. (2009). Publication bias in clinical trials due to statistical significance or direction of trial results. Cochrane Database of Systematic Reviews, Issue 1. Art. No.: MR000006.

Hu, F.B., Goldberg, J., Hedeker, D., Flay, B.R., Pentz, M.A., 1998. Comparison of populationaveraged and subject-specific approaches for analyzing repeated binary outcomes. American Journal of Epidemiology 147, 694-703.

Ioannidis, J. P., Allison, D. B., Ball, C. A., Coulibaly I., Cui X., Culhane, A. C., Falchi, M., Furlanello, C., Game, L., Jurman, G., Mangion, J., Mehta, T., Nitzberg, M., Page, G. P., Petretto, E., van Noort, V. (2009). Repeatability of published microarray gene expression analyses. Nature Genetics. $41: 149-155$

Kilkenny, C., Browne, W.J., Cuthill, I.C., Emerson, M., Altman, D.G., 2010. Improving Bioscience Research Reporting: The ARRIVE Guidelines for Reporting Animal Research. PLoS Biol 8, 1-5. 
655

Kilkenny, C., Parsons, N., Kadyszewski, E., Festing, M.F.W., Cuthill, I.C., Fry, D., Hutton, J., Altman, D.G., 2009. Survey of the Quality of Experimental Design, Statistical Analysis and Reporting of Research Using Animals. PLoS ONE 4, 1-11.

Lahrmann, H.P., Oxholm, L.C., Steinmetz, H., Nielsen, M.B.F., D'Eath, R.B., 2015. The effect of long or chopped straw on pig behaviour. Animal 9, 862-870.

Liao, T.F., 1994. Interpreting probability models: Logit, probit, and other generalized linear models. Sage.

McCance, I., 1995. Assessment of statistical procedures used in papers in the Australian Veterinary Journal. Aus. Vet. J. 72, 322-329.

McGlone, J.J., 1985. A Quantitative Ethogram of Aggressive and Submissive Behaviors in Recently Regrouped Pigs. J. Anim. Sci. 61, 556-566.

Morgan, C.A., Deans, L.A., Lawrence, A.B., Nielsen, B.L., 1998. The effects of straw bedding on the feeding and social behaviour of growing pigs fed by means of single-space feeders. Appl. Anim. Behav. Sci. 58, 23-33.

Munsterhjelm, C., Peltoniemi, O. A., Heinonen, M., Halli, O,. Karhapaa, M., et al. (2009). Experience of moderate "a bedding affects behaviour of growing pigs. Appl Anim Behav Sci. 118:42-53.

Nettle, D., Monaghan, P., Boner, W., Gillespie, R., Bateson, M., 2013. Bottom of the Heap: Having Heavier Competitors Accelerates Early-Life Telomere Loss in the European Starling, Sturnus vulgaris. PLoS ONE 8, e83617.

Sargeant, J.M., Thompson, A., Valcour, J., Elgie, R., Saint-Onge, J., Marcynuk, P., Snedeker, K., 2010. Quality of Reporting of Clinical Trials of Dogs and Cats and Associations with Treatment Effects. J. Vet. Intern. Med. 24, 44-50.

Statham, P., Green, L., Mendl, M., 2011. A longitudinal study of the effects of providing straw at different stages of life on tail-biting and other behaviour in commercially housed pigs. Appl. Anim. Behav. Sci. 134, 100-108.

Taylor, N.R., Main, D.C.J., Mendl, M., Edwards, S.A., 2010. Tail-biting A new perspective. Veterinary Journal 186, 137-147. 
Team, R.C., 2015. R: A Language and Environment for Statistical Computing, R Foundation for 683 Statistical Computing, Vienna, Austria.

684 Touloumis, A., 2016. multgee: GEE Solver for Correlated Nominal or Ordinal Multinomial 685 Responses, http://www.cran.r-project.org/package=multgee.

686 Turner, S.P., Ewen, M., Rooke, J.A., Edwards, S.A., 2000. The effect of space allowance on 687 performance, aggression and immune competence of growing pigs housed on straw deep-litter at 688 different group sizes. Livest. Prod. Sci 66, 47-55.

689 Twisk, J.W.R., 2012. Applied Longitudinal Data Analysis for Epidemiology: A Practical Guide. 2 ed. 690 Cambridge University Press, Cambridge.

691 Vogt L, Reichlin TS, Nathues C, Würbel H (2016) Authorization of Animal Experiments Is Based on 692 Confidence Rather than Evidence of Scientific Rigor. PLoS Biol 14(12): e2000598.

693 Wand, M.P., Jones, M.C., 1994. Kernel Smoothing. Chapman \& Hall/CRC.

694 Wang, Z., Goonewardene, L.A., 2004. The use of MIXED models in the analysis of animal 695 experiments with repeated measures data. Canadian Journal of Animal Science 84, 1-11.

696

697

698

699

700

701

702

703

704

705 
707 Figure 1: The six-point scaling system used to assess injuries to pig's body areas and outline of body areas for injury scoring; Ears, Snout, Shoulders, Legs, Back, Flanks, Hind quarters and Tail.

709 Figure 2: Plots of the log transformed body score by day with a Gaussian kernel smooth estimator with a bandwidth of 15 for a) replication; b) pen; c) enrichment; d) location to the front or back of the experimental room; e) location on either side of the experimental room. The light grey area depicts the time period the second injury assessments were gathered, all points gathered after this period are the third injury assessments and all points before are the first; f) Plot of the pig's relative weight for each pen within replication by log body score with a Gaussian kernel smooth estimator with bandwidth of 4. The grey area of the plot indicates the region where $95 \%$ of the data is located, and where the kernel estimator will be most reliable.

717 Figure 3: a) Box plot of the fixed effect coefficient estimates for the log linear regression model for body score for each replication. The red crosses represent the fixed effect coefficient estimates for the LLME + GEE from table 2. b) Box plot of the fixed coefficient estimates from the ordinal logistic regression of ear score for each replication. The red crosses represent the fixed effect coefficient estimates for the CLME +1 in table 3.cross.

722 Figure 4: Left plots: observed proportion with an ear score of 0 and 1/2. Right plots: observed proportion with an ear score of 0/1 and 2, with Gaussian kernel estimators with a bandwidth of 15 for a) replications; b) pens; c) enrichment; or d) location to the front or the back of the experimental room. The light grey area depicts the time period the second injury assessments were gathered, all injury assessments gathered after this period are the third injury assessments and all injury assessments before are the first. 


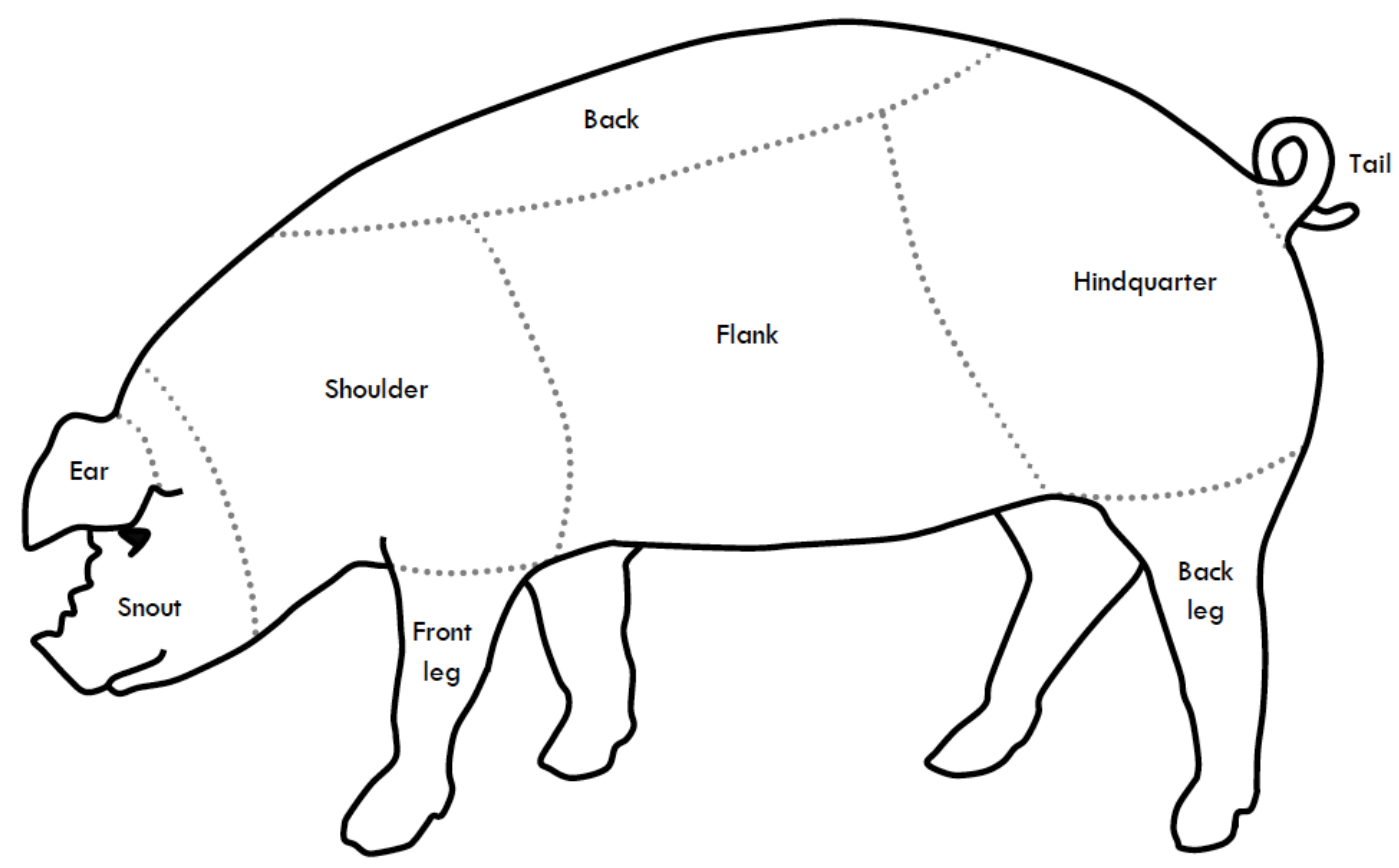

\begin{tabular}{|c|c|}
\hline Score & Scaling System \\
\hline 0 & No injuries. \\
\hline 1 & One small superficial lesion. \\
\hline 2 & $\begin{array}{l}\text { More than one small, superficial lesion; or just one red } \\
\text { (deeper than score 1) but still superficial lesion. }\end{array}$ \\
\hline 3 & $\begin{array}{l}\text { One or several big and deep lesions. If deep, only one } \\
\text { single lesion. If not so deep, several red lesions. }\end{array}$ \\
\hline 4 & $\begin{array}{l}\text { One very big, deep and red lesion. Or many deep, red } \\
\text { lesions. }\end{array}$ \\
\hline 5 & $\begin{array}{l}\text { Many, very big, deep and red lesions covering the skin } \\
\text { area. }\end{array}$ \\
\hline
\end{tabular}



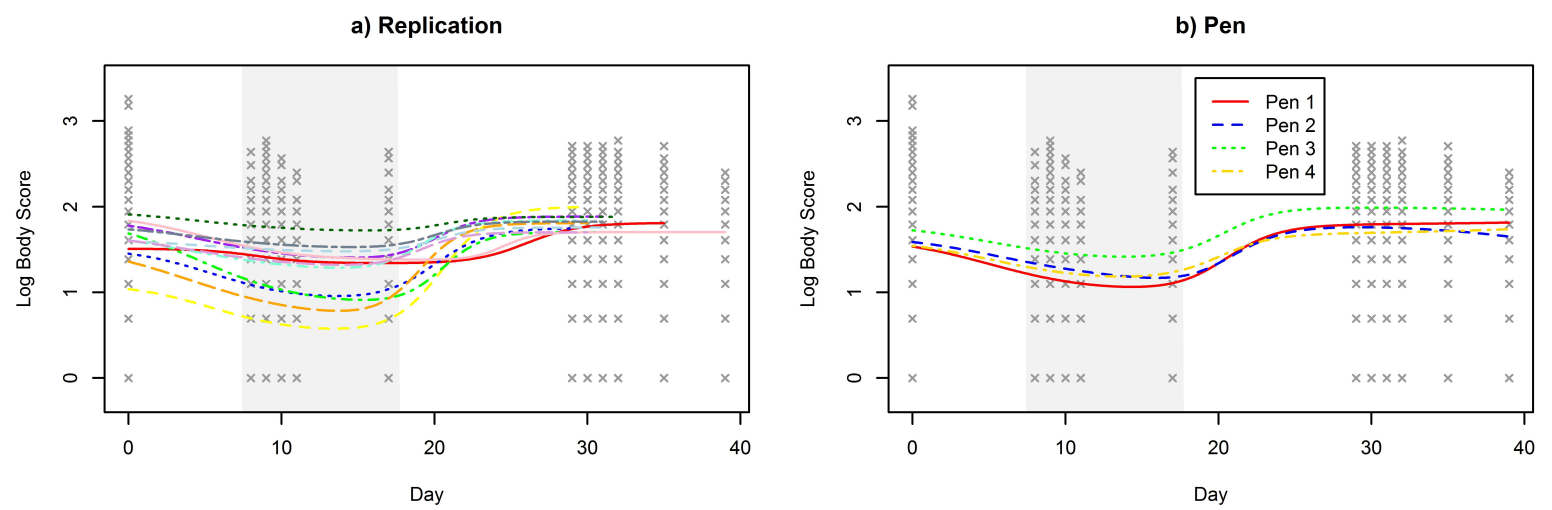

c) Enrichment Level

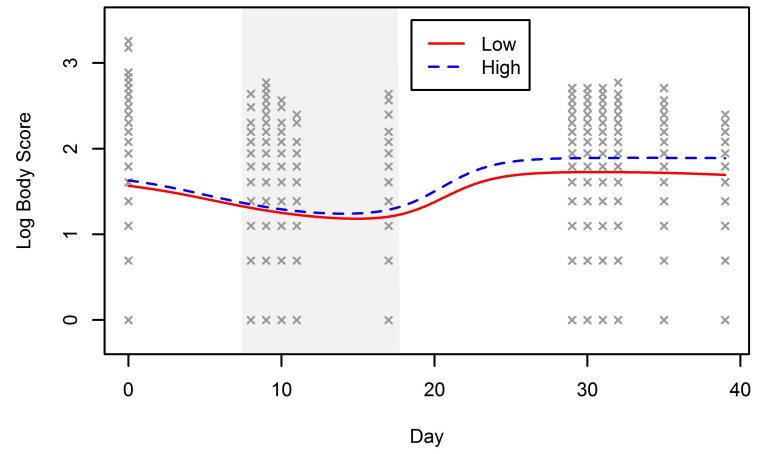

d) Front/Back Location

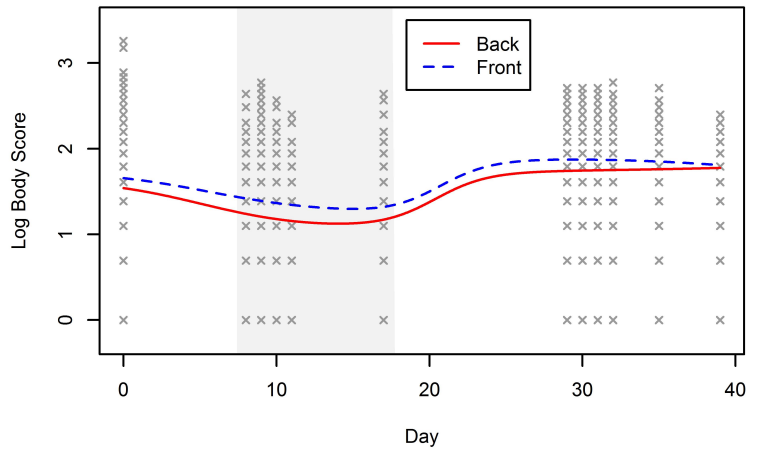

e) Left/Right Side
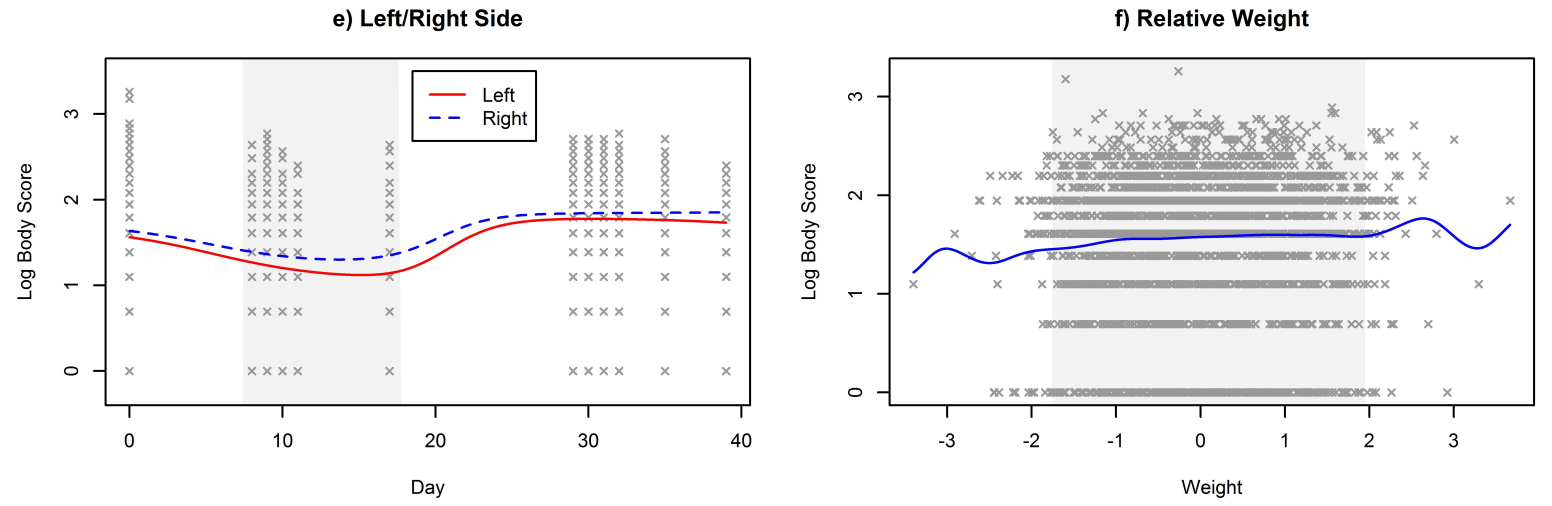
Figure 3
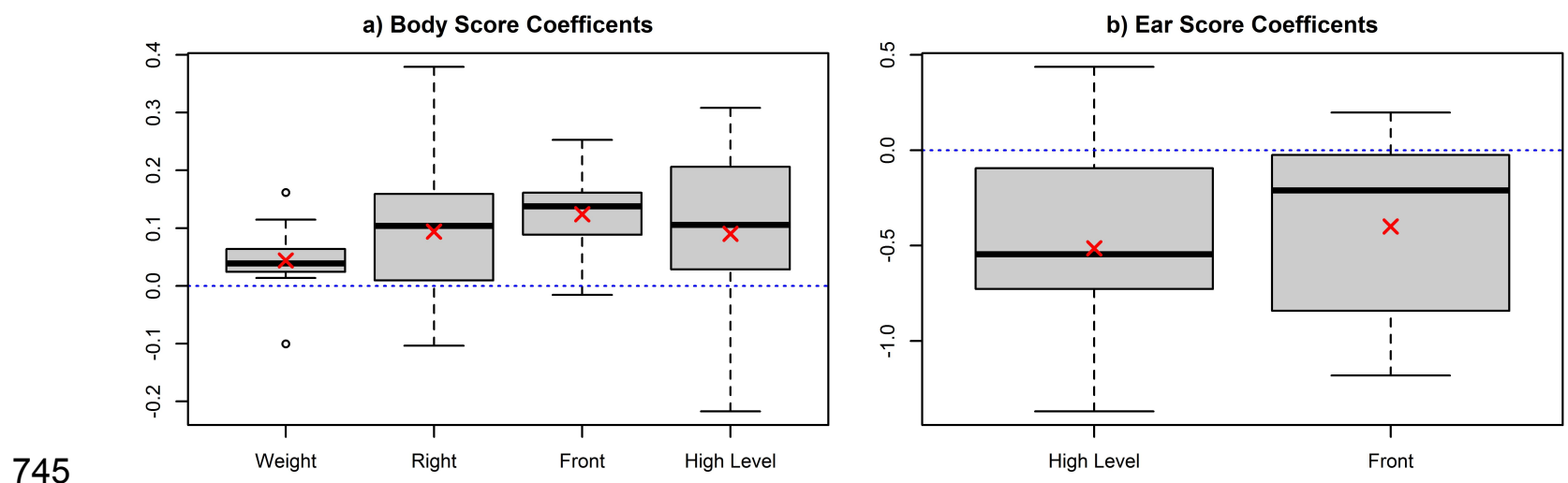

746

747

748

749

750

751

752

753

754

755

756

757

758

759 
a) Replication

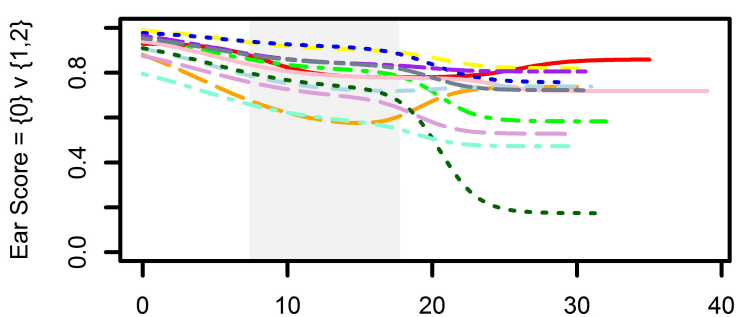

Day Number

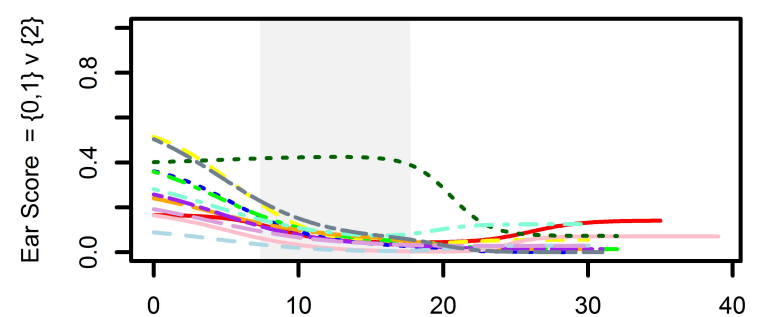

Day Number

b) Pen

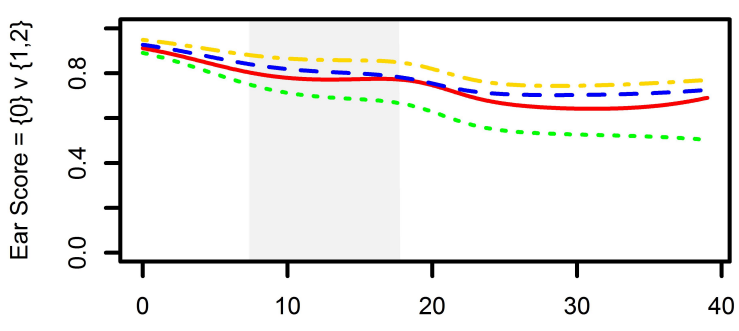

Day Number

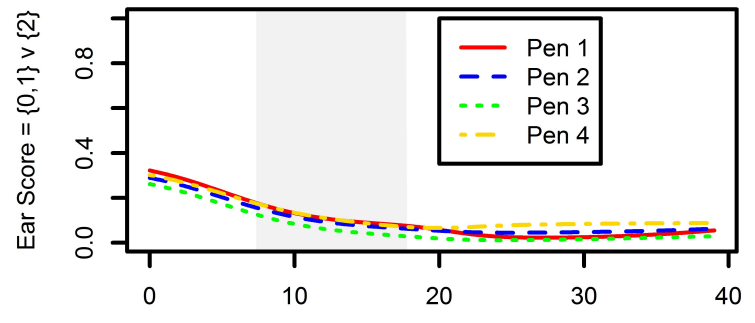

Day Number

c) Enrichment Level
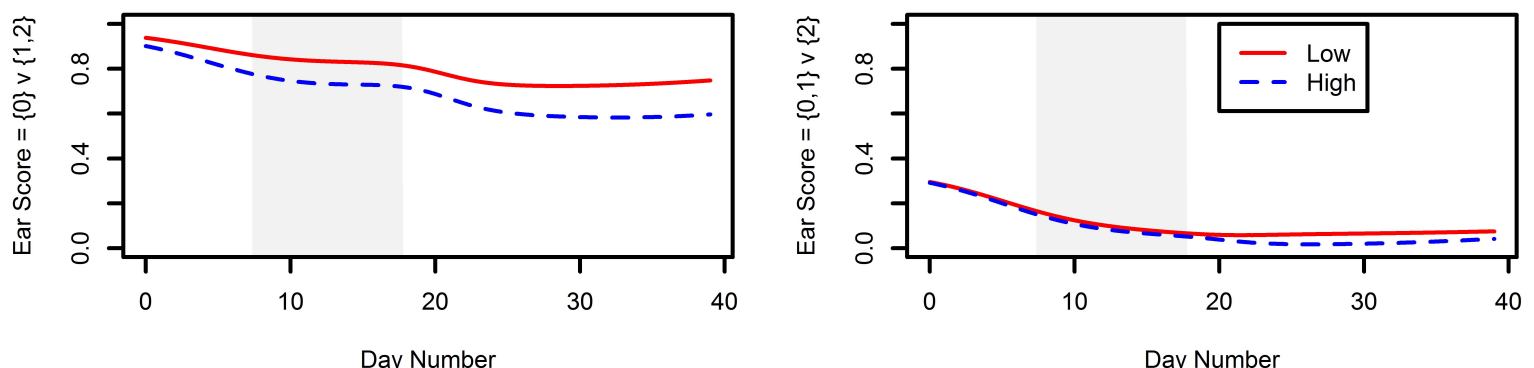

d) Front/Back Location

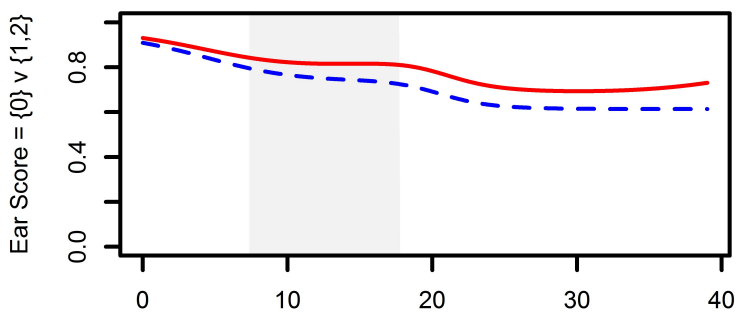

Day Number

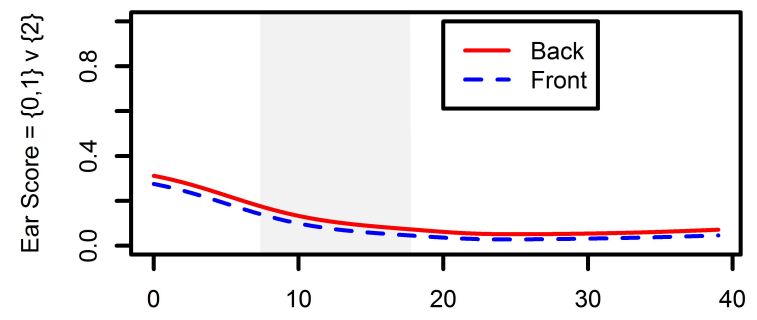

Day Number 


\begin{tabular}{|l|c|c|c|c|c|}
\hline \multirow{2}{*}{ Data } & \multicolumn{5}{|c|}{ Inferential Method } \\
\cline { 2 - 6 } Univariate & MANCOVA & $G L M$ & $L M E$ & $G E E$ & $L M E+G E E$ \\
$\begin{array}{l}\text { Multivariate } \\
\text { Repeated } \\
\text { Hierarchical } \\
\text { Repeated + Hierarchical }\end{array}$ & $\mathrm{C}$ & $\mathrm{C} \mathrm{O}$ & & & \\
\hline
\end{tabular}

Table 1: Types of data that can be analysed using different inference methods, where $\mathrm{C}$ represents continuous data and $\mathrm{O}$ represents ordinal data. MANCOVA=Multivariate Analysis of Covariance; GLM $=$ Generalised linear model; LME=Linear mixed effects model; GEE $=$ General Estimating

770 


\begin{tabular}{|c|c|c|c|c|c|c|c|}
\hline & \multicolumn{4}{|c|}{ LLME + GEE } & LLME & MANCOVA & LLM \\
\hline & & & & $n$ & \multicolumn{3}{|c|}{$n$} \\
\hline Pigs & & & & 862 & 862 & 855 & 862 \\
\hline \multirow[t]{2}{*}{ Body Score } & & & & 2565 & 862 & 2550 & 2556 \\
\hline & $\boldsymbol{\beta}$ & $S E$ & $t$ & $p$ & \multicolumn{3}{|c|}{$p$} \\
\hline Day & 5.87 & 2.47 & 2.38 & 0.0173 & & & $<0.0001$ \\
\hline Day $^{2}$ & 11.45 & 2.35 & 4.87 & $<0.0001$ & & & $<0.0001$ \\
\hline Day $^{3}$ & -6.39 & 1.30 & -4.93 & $<0.0001$ & & & $<0.0001$ \\
\hline More Enriched & 0.09 & 0.04 & 2.40 & 0.0224 & 0.0151 & 0.0003 & 0.0003 \\
\hline Location: Right & 0.08 & 0.04 & 2.26 & 0.0307 & 0.0109 & 0.0018 & $<0.0001$ \\
\hline Sex & & & & & & 0.0041 & \\
\hline Weight & 0.05 & 0.01 & 3.41 & 0.0007 & & 0.0278 & 0.0013 \\
\hline Location: Front & 0.11 & 0.04 & 3.16 & 0.0034 & 0.0011 & 0.0003 & $<0.0001$ \\
\hline
\end{tabular}

Table 2: Summary statistics for inferential analysis of Body Score via the: log linear mixed effects model for repeated measures (LLME + GEE); linear mixed effects model of pig's mean log body score (LME); multivariate analysis of covariance (MANCOVA) of log body score, and a log linear regression model (LLM). Where: $\boldsymbol{n}$ is the number of pigs/body score assessment; $\boldsymbol{\beta}$ is the parameter estimate; $\boldsymbol{S E}$ is the standard error; $\boldsymbol{t}$ is the Student's t test statistic and $\boldsymbol{p}$ is the probability value associated with each covariate. Day is the day within the trial that observations were recorded; More Enriched refers to pens that had more enrichment (compared with Less Enriched); Location: Right refers to pens on the right side of the room (compared to pens on the left side of the room); Location: Front refers to pens at the front of the room (compared to pens at the back of the room).

791 


\begin{tabular}{|c|c|c|c|c|c|c|c|}
\hline & \multicolumn{4}{|c|}{ CLME + 1} & CLME & GEE & CLM \\
\hline & & & & $n$ & \multicolumn{3}{|c|}{$n$} \\
\hline Pigs & & & & 862 & 862 & 862 & 862 \\
\hline \multirow[t]{2}{*}{ Ear Score } & & & & 2572 & 862 & 2572 & 2572 \\
\hline & $\boldsymbol{\beta}$ & $S E$ & $t$ & $p$ & \multicolumn{3}{|c|}{$p$} \\
\hline Day & -51.68 & 5.75 & -8.99 & $<0.0001$ & & $<0.0001$ & $<0.0001$ \\
\hline Day $^{2}$ & 31.30 & 5.74 & 5.45 & $<0.0001$ & & $<0.0001$ & $<0.0001$ \\
\hline Day $^{3}$ & -13.56 & 6.51 & -2.08 & $<0.0369$ & & 0.0453 & 0.0003 \\
\hline Day $^{4}$ & & & & & & $<0.0001$ & $<0.0001$ \\
\hline Day $^{5}$ & & & & & & 0.0194 & $<0.0001$ \\
\hline Day $^{6}$ & & & & & & & 0.0255 \\
\hline Day $^{7}$ & & & & & & & $<0.0001$ \\
\hline More Enriched & -0.51 & 0.18 & -2.79 & 0.0053 & 0.0131 & $<0.0001$ & $<0.0001$ \\
\hline Weight & & & & & 0.0302 & & \\
\hline Location: Front & -0.40 & 0.18 & -2.25 & 0.0247 & 0.0328 & $<0.0001$ & $<0.0001$ \\
\hline
\end{tabular}

Table 3: Summary statistics for inferential analysis of Ear Score via the: cumulative logistic mixed

798 effects model with rep, pen and pig random effects (CLME +1$)$; cumulative logistic mixed effects

799 model with rep and pen random effects for summary ear score (CLME); cumulative logistic

800 regression model for repeated measures (GEE); the cumulative logistic regression model (CLM).

801 Where: $\boldsymbol{n}$ is the number of pigs/ear score assessment; $\boldsymbol{\beta}$ is the parameter estimate; $\boldsymbol{S} \boldsymbol{E}$ is the standard 802 error; $\boldsymbol{t}$ is the Student's t test statistic and $\boldsymbol{p}$ is the probability value associated with each covariate.

803 Day is the day within the trial that observations were recorded; More Enriched refers to pens that 804 had more enrichment (compared with Less Enriched); Location: Front refers to pens at the front of 805 the room (compared to pens at the back of the room). 